\title{
A qPCR to quantify Wolbachia from few Onchocerca volvulus microfilariae as a surrogate for adult worm histology in clinical trials of antiwolbachial drugs
}

\author{
Stefan Schlabe ${ }^{1,2,3} \cdot$ Patricia Korir $^{2,3} \cdot$ Christine Lämmer $^{2,3}$. Frederic Landmann ${ }^{4} \cdot$ Bettina Dubben $^{2,3}$. \\ Marianne Koschel $^{2,3} \cdot$ Anna Albers $^{2,3} \cdot$ Linda Batsa Debrah ${ }^{5,6} \cdot$ Alexander Yaw Debrah $^{5,7}$ - Marc P. Hübner ${ }^{2,3}$. \\ Kenneth Pfarr ${ }^{2,3}$ (1) Ute Klarmann-Schulz ${ }^{2,3} \cdot$ Achim Hoerauf ${ }^{2,3}$
}

Received: 30 August 2021 / Accepted: 14 December 2021 / Published online: 10 January 2022

(c) The Author(s) 2022

\begin{abstract}
The filarial nematode Onchocerca volvulus causes onchocerciasis (river blindness), a neglected tropical disease affecting 21 million people, mostly in Sub-Saharan Africa. Targeting the endosymbiont Wolbachia with antibiotics leads to permanent sterilization and killing of adult worms. The gold standard to assess Wolbachia depletion is the histological examination of adult worms in nodules beginning at 6 months post-treatment. However, nodules can only be used once, limiting the time points to monitor Wolbachia depletion. A diagnostic to longitudinally monitor Wolbachia depletion from microfilariae (MF) at more frequent intervals $<6$ months post-treatment would accelerate clinical trials of antiwolbachials. We developed a TaqMan qPCR amplifying the single-copy gene $w \mathrm{Ovfts} Z$ to quantify Wolbachia from as few as one MF that had migrated from skin biopsies and compared quantification using circular and linearized plasmids or synthetic dsDNA (gBlock®). qPCR for MF from the rodent nematode Litomosoides sigmodontis was used to support the reproducibility and validate the principle. The qPCR using as few as $2 \mathrm{MF}$ from $O$. volvulus and L. sigmodontis reproducibly quantified Wolbachia. Use of a linearized plasmid standard or synthesized dsDNA resulted in numbers of Wolbachia/MF congruent with biologically plausible estimates in $O$. volvulus and $L$. sigmodontis MF. The qPCR assay yielded a median of 48.8 (range 1.5-280.5) WolbachialO. volvulus MF. The qPCR is a sensitive tool for quantifying Wolbachia in a few MF from skin biopsies and allows for establishing the qPCR as a surrogate parameter for monitoring Wolbachia depletion in adult worms of new antiwolbachial candidates.
\end{abstract}

Keywords qPCR $\cdot$ Wolbachia $\cdot$ Onchocerca volvulus $\cdot$ Litomosoides sigmodontis

Section Editor: Christoph G. Grevelding

Ute Klarmann-Schulz and Achim Hoerauf shared last author

Kenneth Pfarr

Kenneth.Pfarr@ukbonn.de

1 Department of Internal Medicine I, University Hospital Bonn, Bonn, Germany

2 German Center for Infection Research (DZIF), Partner Site Bonn-Cologne, Bonn, Germany

3 Institute of Medical Microbiology, Immunology and Parasitology, University Hospital Bonn, Bonn, Germany

4 Centre de Recherche de Biologie Cellulaire de Montpellier (CRBM), Université de Montpellier, CNRS, 34293 Montpellier, France
5 Kumasi Centre for Collaborative Research in Tropical Medicine (KCCR), Kumasi, Ghana

6 Department of Clinical Microbiology, Kwame Nkrumah University of Science and Technology, Kumasi, Ghana

7 Faculty of Allied Health Sciences of Kwame, Nkrumah University of Science and Technology, Kumasi, Ghana 


\section{Introduction}

Onchocerca volvulus nematodes cause onchocerciasis, a neglected tropical disease (NTD) known as river blindness, affecting 21 million people in Sub-Saharan Africa and remaining foci in Latin America. While Mass Drug Administration (MDA) was established to reduce transmission by targeting microfilariae (MF), elimination may not be possible for all endemic areas using MDA alone due to persisting, long-lived adult worms and reported suboptimal responses (Turner et al. 2013; WHO 2020). To achieve the WHO elimination goals by 2030 , medicines that permanently sterilize or kill adult worms and sensitive diagnostics are needed. To address the first, antiwolbachial therapies, e.g., doxycycline, targeting the essential Gram-negative Wolbachia endosymbionts, provide an effective tool, preventing microfilariae (MF)-deathinduced inflammation and adverse events and killing adult filariae within 2 years (Debrah et al. 2015; Hoerauf et al. 2001; Hoerauf et al. 2008; Klarmann-Schulz et al. 2017). To overcome the long treatment time with doxycycline (4-6 weeks), new compounds have been sought and several are entering phase 1 and 2 trials (Hübner et al. 2019, 2020; Krücken et al. 2021; Taylor et al. 2019; von Geldern et al. 2019; Hong et al. 2019). Even with nucleic acid amplification tests, one major obstacle to the development of new antiwolbachial drugs is that the treatment protocols lack an adequate early endpoint monitoring Wolbachia reduction necessary for go/no-go decisions. A significant effect on the adult worms can first be evaluated by histology after 18-24 months (Debrah et al. 2015; Hoerauf et al. 2008), with Wolbachia depletion preceding the macrofilaricidal effect (Hoerauf et al. 2001, 2008), a parameter analyzed together with the adult worm viability and therefore usually performed at the primary study endpoint (Klarmann-Schulz et al. 2017). Moreover, diagnostics targeting adult worms rely on surgical extirpation of the nodules, limiting the number of time points that can be examined. A diagnostic tool using MF emerged from skin snips would enable frequent and longitudinal monitoring and evaluation of the dynamics of the Wolbachia depletion.

We developed a qPCR protocol diagnostic tool for absolute quantification of Wolbachia in few MF by using commercially manufactured DNA fragments (gBlocks ${ }^{\circledR}$ ) as qPCR standards. The qPCR allows longitudinal monitoring of Wolbachia at timepoints from treatment start to nodule extirpation and can be used to evaluate early treatment effect in clinical trials of antiwolbachial treatments.

\section{Material and methods}

\section{Samples}

O. volvulus MF were collected from MF that had migrated out of skin snips (Hoerauf et al. 2008). MF were counted in 24-well plates in which the initial DNA extraction step was also performed. DNA was extracted using a modification of the Qiagen DNA QiaAmp Micro Kit protocol using a minimum elution volume of $25 \mu \mathrm{L}$ that was incubated on the column twice for $5 \mathrm{~min}$. The samples were derived from three different antiwolbachial clinical trials (DOLF, ISRCTN50035143 (Batsa Debrah et al. 2020); MoRiOn, ISRCTN43697583; and AWOL-Mino, ISRCTN06010453 (Klarmann-Schulz et al. 2017)). An MF positive control to calculate the intraassay and interassay variability and stability after long-term storage was made by pooling 2517 MF from 11 archived samples from untreated participants (DOLF, ISRCTN50035143 (Batsa Debrah et al. 2020)).

\section{qPCR assay}

The qPCR amplifies the single-copy gene fts $Z$ from Wolbachia (wOvftsZ; GenBank:AJ276501) and actin from $O$. volvulus (OvActin; GenBank:M84916.1). For standardization studies and technical performance analyses, we used a duplex qPCR for MF from the rodent filarial nematode L. sigmodontis (Hübner et al. 2019). Both qPCRs used TaqMan probes. Standard curves were created using a 1:10 dilution series of the mixed standards from $10^{7}$ copies/ $\mu \mathrm{L}$ to $10^{0}$ copies $/ \mu \mathrm{L}$. All primers were synthesized by Microsynth AG, Balgach, Switzerland; TaqMan probes were synthesized by Biomers GmbH, Ulm, Germany (Table 1). Absolute quantification was done as a duplex qPCR with a reaction volume of $20 \mu \mathrm{L}$ in a RotorGene 6000 (Qiagen, Hilden, Germany): 1xQuantiNova master mix (Qiagen), $400 \mathrm{nM}$ for each forward and reverse primer, and $25 \mathrm{nM}$ and $50 \mathrm{nM}$ hybridization probe for $w \mathrm{Ovfts} Z$ and OvActin, respectively, with activation at $95{ }^{\circ} \mathrm{C}$ for $2 \mathrm{~min}$, followed by 45 cycles of $95{ }^{\circ} \mathrm{C}$ for $5 \mathrm{~s}$ and amplification at $58{ }^{\circ} \mathrm{C}$ for $30 \mathrm{~s}$ with fluorescence acquired on FAM (green) and JOE (yellow) channels at $58{ }^{\circ} \mathrm{C}$. All samples were measured in triplicate. Analysis of qPCR data was done using the RotorGene 6000 Series Software ver. 1.7 with normalization on control reactions (no-template controls, NTC). Quantification was determined by setting the fluorescence threshold at 0.01 . Outlier removal was set to $15 \%$ (only signals $15 \%$ higher than background were considered true).

Plasmid standards containing the $w \mathrm{Ovfts} Z$ sequence were tested in two different conformations: as supercoiled plasmids and after linearization with the singlecutting restriction NotI-HF enzyme according to the manufacturer's protocol (NEB, Ipswich, MA). For absolute quantification, we designed artificial dsDNA fragments (gBlock ${ }^{\circledR}$, Integrated DNA Technologies, Coralville, Iowa, USA) (Table 1), dissolved in AE buffer to yield a final concentration of $1.2 * 10^{10}$ copies $/ \mu L$. For 
Table 1 Primers, probes, and gBlock® sequences

\begin{tabular}{|c|c|c|}
\hline Primers & $5^{\prime}-3^{\prime}$ & $\mathrm{Tm}^{\circ} \mathrm{C}$ \\
\hline$w \mathrm{OvftsZ \textrm {FW }}$ & AGGAATGGGTGGTGGTACTG & 60.5 \\
\hline$w O v f t s Z R V$ & CTTTAACCGCAGCTCTTGCT & 58.4 \\
\hline OvActin FW & GTGCTACGTTGCTTTGGACT & 58.4 \\
\hline OvActin RV & GTAATCACTTGGCCATCAGG & 58.4 \\
\hline$w \mathrm{LsftsZ \textrm {FW }}$ & CGATGAGATTATGGAACATATAA & 55.6 \\
\hline$w \operatorname{LsftsZ}$ & TTGCAATTACTGGTGCTGC & 55 \\
\hline LsActin FW & ATCCAAGCTGTCCTGTCTCT & 58.4 \\
\hline LsActin RV & TGAGAATTGATTTGAGCTAATG & 54.8 \\
\hline Probes & $5^{\prime}-3$ & \\
\hline$w \mathrm{OvftsZTQP}$ & 6-FAM CCTTGCCGCTTTCGCAATCAC DDQ1 & 61 \\
\hline OvActinTQP & HEX AACAGGAAATGGCAACTGCTGC BHQ-1 & 58 \\
\hline$w$ LsftsZ TQP & 6-FAMCAGGGATGGGTGGTGGTACTGGAA TAMRA & 63 \\
\hline LsActinTQP & HEX ACTACCGGTATTGTGCTCGATT TAMRA & 52 \\
\hline \multicolumn{3}{|l|}{ gBlock $®$} \\
\hline$w O v f t s Z$ & $\begin{array}{l}\text { CATATAAAAGATAGTCATATGCTCTTTATTACAGCAGGAAT } \\
\text { GGGTGGTGGTACTGGTACAGGTGCAGCACCAGTGATTG } \\
\text { CGAAAGCGGCAAGGGAAGCAAGAGCTGCGGTTAAAGAT } \\
\text { AAAATGTTAAAAGAGAAAAAGATATTGACTGTT }^{\#}\end{array}$ & \\
\hline OvActin & $\begin{array}{l}\text { TTGTTCGTGACATCAAAGAAAAGCTGTGCTACGTTGCTTT } \\
\text { GGACTTCGAACAGGAAATGGCAACTGCTGCATCGTCATC } \\
\text { GTCTCTCGAAAAATCTTATGAATTGCCTGATGGCCAAGT } \\
\text { GATTACCGTAGGCAACGAACGATTTCGATGCC }\end{array}$ & \\
\hline
\end{tabular}

\#Target sequence is in bold. The underlined sequences are flanking regions from the genomic sequence to reach the $\mathrm{gBlock} \circledast$ minimum length of $150 \mathrm{bp}$ quantification assays, a standard curve of $1.2 * 10^{5}$ to $1.2 * 10^{0}$ copies/ $\mu \mathrm{L}$ was used.

\section{Immunofluorescence picture of $L$. sigmodontis MF}

To determine the number of Wolbachia in a single MF, we fixed and stained L. sigmodontis MF (Landmann et al. 2012). The Wolbachia in the MF were labeled with anti-Wolbachia Surface Protein monoclonal antibody (1:500, NR-31029, obtained through BEI Resources, NIAID, NIH) (Fattouh et al. 2019) and detected with a rabbit anti-mouse antibody tagged with Alexa fluor $488 \mathrm{~nm}$ (Invitrogen, 1:500). MF nuclei were stained with DAPI (Serbus et al. 2012). MF were observed with a $63 \mathrm{X}$ objective on a Leica SP5 confocal microscope.

\section{Results and discussion}

\section{Linear plasmid standards are required for absolute quantification of Wolbachia in L. sigmodontis MF}

We established absolute quantification of Wolbachia per MF using L. sigmodontis MF. Parallel qPCR runs of NotI linearized and circular plasmid standards resulted in a tenfold difference in Wolbachia number, with $20.6 \mathrm{wLs} f t \mathrm{ZI}$ MF calculated using a linearized plasmid vs. $200 \mathrm{wLsftsZ/}$ MF when using the circular (Fig. 1A). The difference in Wolbachia numbers was most likely due to less efficient amplification using supercoiled plasmid standards (Hou et al. 2010; Lin et al. 2011). To support that the linearized plasmid standard curve resulted in the biologically relevant number of Wolbachia in an MF, we compared the numbers provided by the qPCR with immunofluorescence images of L. sigmodontis MF (Fig. 1B). Using immunofluorescence, the MF clearly contained fewer than 200 Wolbachia. Thus, we concluded that the quantification using the linearized plasmid was more accurate.

To assess the performance of the qPCR on samples with different MF counts, including only a few MF, the DNA extraction and qPCR were systematically analyzed with duplicate samples of 5 to $60 \mathrm{MF}$. wLsfts $Z$ copy number varied in samples with low MF counts, whereas samples with $>20 \mathrm{MF}$ had calculated values within the $95 \%$ confidence bars (Fig. 1C). Nevertheless, all calculated Wolbachia numbers were within a biologically realistic range (5-39). In 3 independent extractions of MF from the same animal, the qPCR had good interassay reliability of Wolbachial 
A

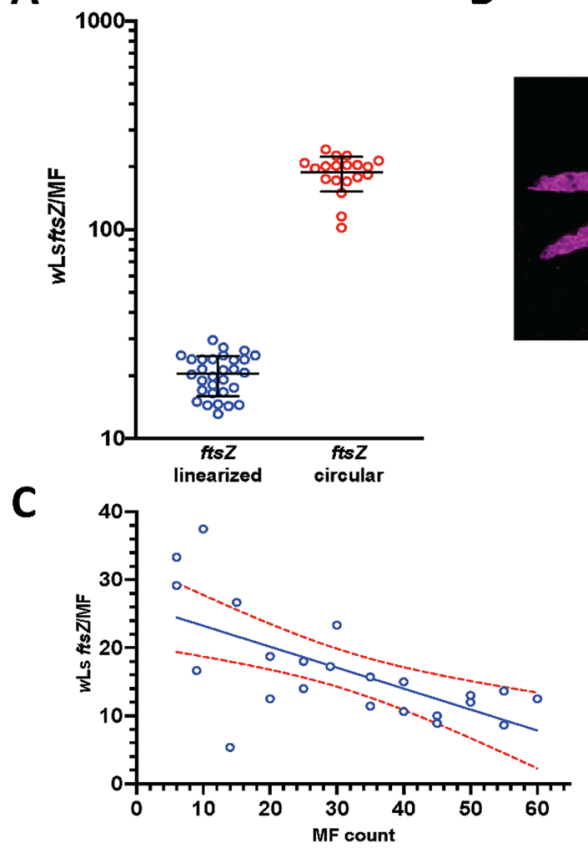

Fig. 1 Supercoiled plasmid standards overestimate $w$ Lsfts $Z$ copy numbers. (A) L. sigmodontis MF were counted (range 17 to 600 ) and DNA extracted for $w$ LsftsZ qPCR. Quantification was done using circular $(N=19)$ or NotI linearized $(N=30)$ plasmids. Linearized plasmids resulted in reduced copy numbers (20.6 vs. $200 \mathrm{wLsftsZ/MF).}$ Lines indicate median and interquartile range. (B) Full projection of confocal images showing 2 Litomosoides sigmodontis microfilariae stained with DAPI (magenta) and an anti-WSP (yellow) monoclonal antibody. Clusters of Wolbachia are located in the proximal part of the posterior half of MF. A single Wolbachia bacterium is $0.8-1 \mu \mathrm{m}$ in length; thus, there are not hundreds of endobacteria in a single MF.

MF counts with a median between 14 and 25 (range 8-28) (Fig. 1D). The interassay reliability was confirmed using a second MF population (Supplementary Table 1).

\section{O. volvulus Wolbachia qPCR in MF is a surrogate for adult worm histology}

Operable onchocercomata are a limited resource in drug trials, require invasive operations, and can be performed infrequently to provide Wolbachia histology, usually at the start and end of the study. The main objective in the establishment and validation of the qPCR was, therefore, the generation of a reliable method to assess Wolbachia reduction early in clinical trials that can be easily and frequently collected without the need for surgically obtained adult worms. Skin snips, which are less invasive and logistically easier to perform, can be taken at many timepoints throughout a study to monitor Wolbachia densities in MF (e.g., pre-treatment, during treatment, and every 2 months post-treatment followup) and generate robust data on the dynamics of Wolbachia depletion and possible rebounds when applying suboptimal

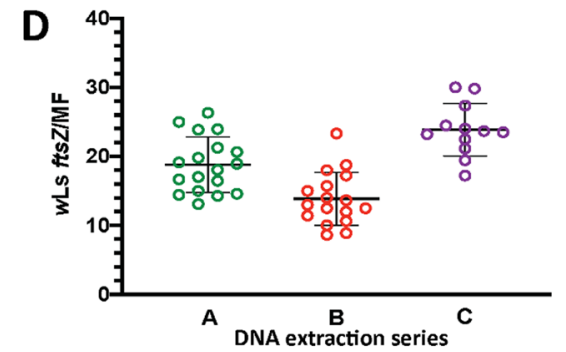

MF were observed with a $63 \mathrm{X}$ objective on a Leica SP5. The scale bar $=10 \mu \mathrm{M}$. (C) Variation in $w$ Lsfts $Z$ copy number per L. sigmodontis $\mathrm{MF}$ is greater in samples with few MF. DNA was extracted from 5 to $60 \mathrm{~L}$. sigmodontis $\mathrm{MF}$ and used for $w \mathrm{LsftsZ} \mathrm{qPCR}$. Linear regression (blue line) and 95\% confidence bars (red dotted lines) are shown. (D) The qPCR is reproducible and stable. Interassay reproducibility was tested on DNA extracted from 18 preparations, each with (A) 50 $\mathrm{MF}$ and (B) $20 \mathrm{MF}$, and (C) 12 preparations with $50 \mathrm{MF}$ from $\mathrm{L}$. sigmodontis to calculate $w \mathrm{Lsfts} Z$ copies/MF. Lines indicate median and interquartile range

treatments. In prior trials, histology of nodules was done at 6 months, 20 months, and 27 months after therapy, and it was shown that the Wolbachia depletion in adult worms seen at 20 months was already established at month 6 (ISRCTN71141922 and ISRCTN68861628) (Batsa-Debrah et al. 2017; Hoerauf et al. 2008; Specht et al. 2009). It must, however, be stated that while Wolbachia reduction by $2 \operatorname{logs}$, in past trials, has always preceded the treatment success confirmed by histology of the adult worms, parasitological success cannot be predicted definitely by Wolbachia depletion at months 6 or earlier, as these numbers may later rebound if treatment has been suboptimal. Based on human and animal trials, a sustained Wolbachia depletion by a threshold of at least $2 \log$ s is required for female adult worm sterilization and macrofilaricidal effects (Albers 2011; Hong et al. 2019; Hübner et al. 2019; Specht et al. 2018; Turner et al. 2006).

To correlate Wolbachia/MF with the antiwolbachial effect in the adult worms, we measured the $w \mathrm{Ovfts} Z$ levels in $\mathrm{MF}$ and compared the result to the Wolbachia levels in the adults assessed by histology in a recent trial on different regimens with doxycycline and rifampicin (ISRCTN06010453 
(Klarmann-Schulz et al. 2017)). The Wolbachia depletion from adult worms had been determined by histology at 6 months after treatment in that trial. The Wolbachia levels of the MF from adult worms were divided into four groups: dead worms and many, few or no Wolbachia. The qPCR was done on DNA extracted from MF collected 6 months after treatment. The Wolbachia/MF determined by qPCR significantly correlated with groups of many, few, and no Wolbachia (Fig. 2). There was a significant difference between the four groups regarding the Wolbachia $w \mathrm{OvFtsZ/}$ MF ( $p=0.003$, Kruskal-Wallis test) with the main differences between many (median 332 wOvftsZ copies/MF; IQR 128,2665 ) and no Wolbachia (median $1 \mathrm{w}$ Ovfts $Z$ copies/ MF; IQR 1, 20; $p=003$, Mann-Whitney $U$-test) as well as between many (median 332 wOvfts $Z$ copies/MF; IQR 128,2665 ) and few Wolbachia (median 1 wOvfts $Z$ copies/ MF; IQR 1, 193; $p=0.004$, Mann-Whitney $U$-test). Thus, 6 months after treatment, the MF qPCR confirmed the Wolbachia depletion from adult worms determined by histology, validating the quantification of Wolbachia depletion from MF from skin snips as a suitable surrogate parameter

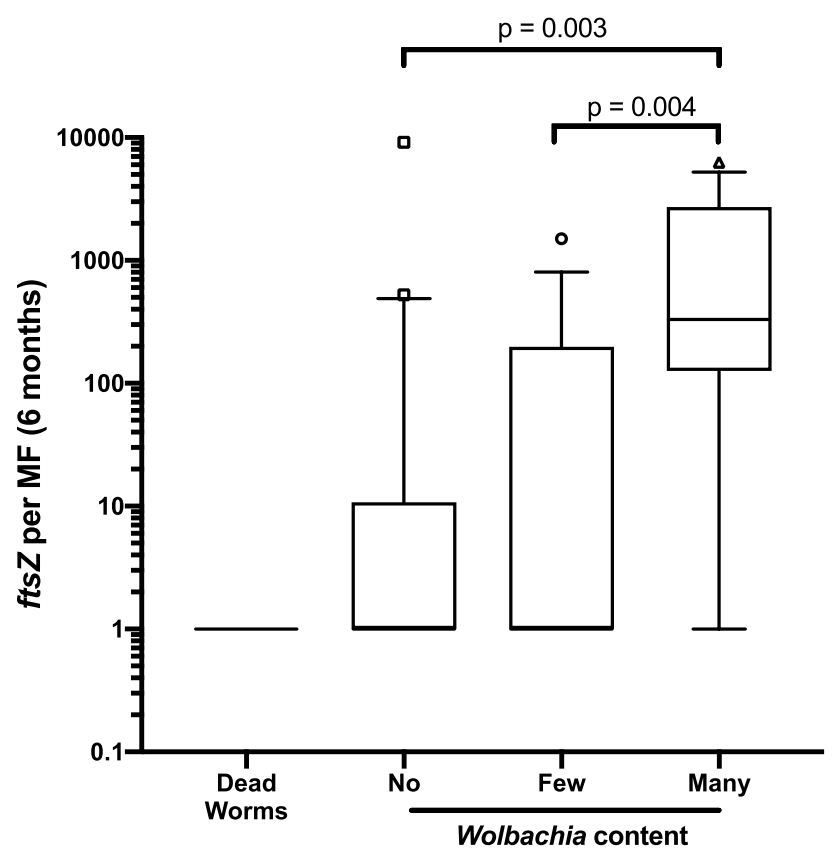

Fig. 2 wOvftsZ Wolbachia qPCR of MF isolated from skin snips correlates with histological assessment of Wolbachia content in adult worms. The Wolbachia levels in adult worms at 6 months after treatment were divided into three classifications (no $(N=20)$, few $(N=17)$, many $(N=12)$ Wolbachia or 1 dead worm) by histology. $w O v f t s Z$ was measured by qPCR in the MF that had emerged from skin snips taken from the corresponding patients in the 6 months follow-up. There was a significant difference between the four groups regarding the Wolbachia $w \mathrm{OvFtsZ/MF} \mathrm{(} p=0.003$, Kruskal-Wallis test) with the main differences between many and no Wolbachia as well as many and few Wolbachia $(p=0.003, p=0.004$, respectively, Mann-Whitney $U$-test, SPSS version 24 for adult worm histology so that nodulectomies at this time point can be spared.

\section{The 0 . volvulus Wolbachia qPCR is sensitive for absolute quantification and longitudinal monitoring}

Similar to L. sigmodontis, the copy number calculations done with the circular plasmid standard resulted in implausibly high Wolbachia counts in $O$. volvulus, i.e., several thousand Wolbachia per MF (Fig. 2). Such numbers were never seen by immunohistology and electron microscopy (Büttner et al. 2003; Martinez-Palomo and Martinez-Baez 1977). Nevertheless, the qPCR assay had a low variation of just $1 \mathrm{Ct}$ in 67 runs performed over one year using aliquots of the same positive control sample (Fig. 3A). Use of NotI linearized plasmid as the template for a standard curve resulted in a shift in $\mathrm{Ct}$ of 3 cycles leading to eightfold lower $w \mathrm{Ovfts} Z$ copy number compared to circularized plasmids (Fig. 3B, Supplementary Fig. 1). Attempts were made to do immunofluorescence in $O$. volvulus $\mathrm{MF}$, but the fixation in ethanol in the field did not allow for good freeze-cracking and subsequent staining with the anti-WSP antibody.

To remove errors introduced by the preparation of plasmid DNA as standards, e.g., errors introduced by method of DNA quantification (Supplementary Fig. 2), the target sequences were synthesized as gBlock ${ }^{\circledR}$ (Conte et al. 2018). Because the gBlock $®$ concentration was determined by HPLC, the numbers calculated from this standard are more accurate than other methods of DNA quantification. The reliable limit of detection was $10 w \mathrm{Ov} f t s Z / \mu \mathrm{L}$ (Supplementary Table 2). The qPCR could detect as few as 1 $w \mathrm{Ov} f t s Z / \mu \mathrm{L}$, but the variation of the triplicates was too great or not all replicates gave a signal or were close to background levels with $\mathrm{Ct}$ near 35 (Supplementary Table 2). The assay using gBlocks ${ }^{\circledR}$ was performed on 34 samples with a range of MF counts (1 to 693) per DNA extraction. This resulted in a median $48.8 \mathrm{wOvftsZ/MF}$ (range 1.5-280.5, IQR 117.6) (Fig. 3C)).We concluded that the copy number calculated from the linearized plasmid/gBlock ${ }^{\circledR}$ was correct and was $<200$ Wolbachia/MF. In the same sample set, the median actin copy number calculation was 1241.4 copies/MF (range 169.2-4111.8, IQR 1087.9). We do not know the number of cells per MF; an estimation can be 500 diploid cells. The median actin copy number calculation of our qPCR is biologically plausible, as the actin primers and probe will amplify both actin 1 and 2 . The variation of the Wolbachia densities in this set of MF samples is obvious. We assume that the major contribution to this variation is caused by biological variation, different Wolbachia numbers in MF populations, which vary in adult worm age.

The $w$ OvftsZ/MF variation was larger when using DNA extracted from low numbers of MF, in which one of three 

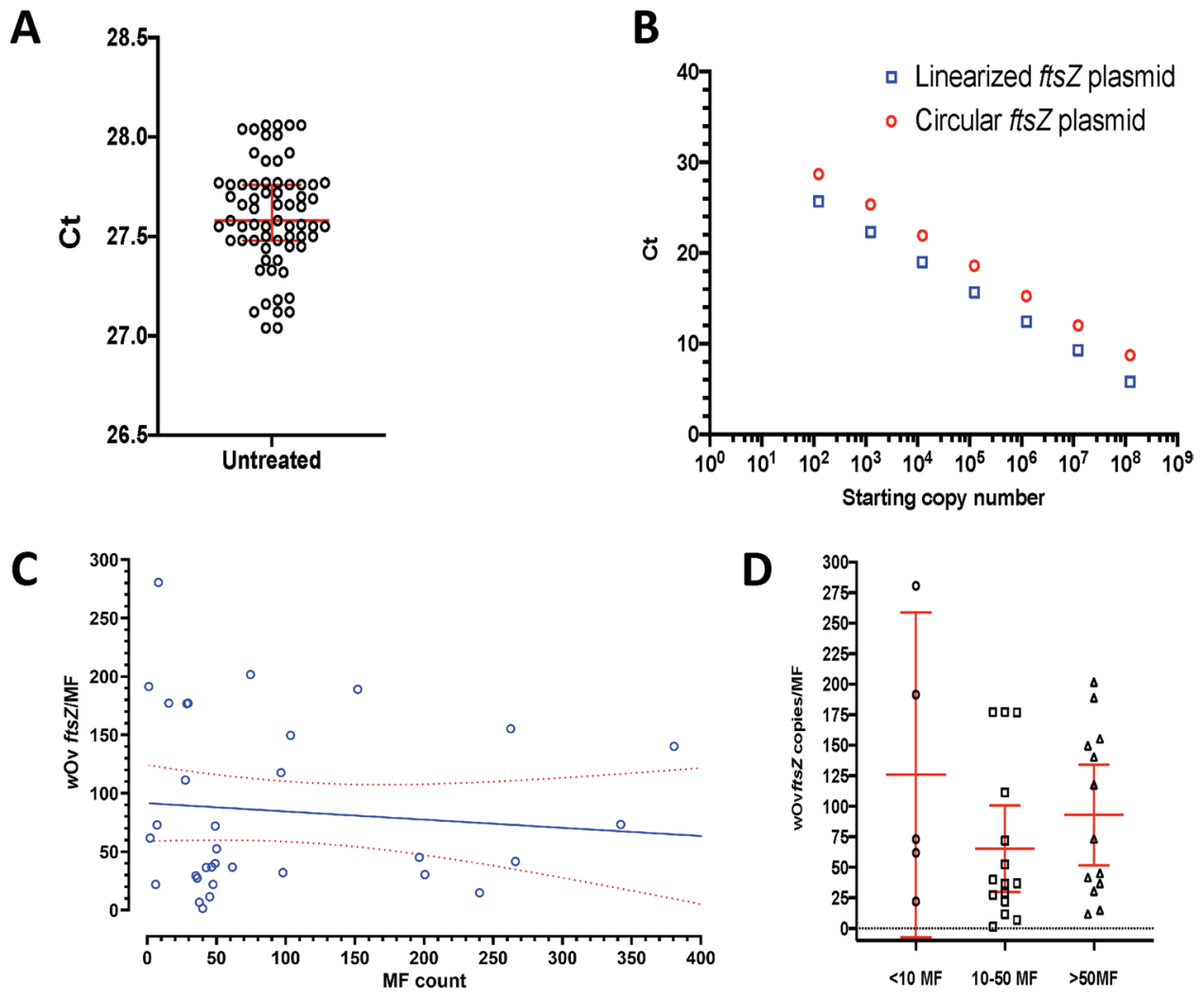

Fig. 3 qPCR on $O$. volvulus Wolbachia show reliable quantification with linearized DNA standards. (A) Technical reproducibility of qPCR was performed using a positive control sample containing DNA from pooled MF samples from untreated patients, 67 qPCR runs were performed over a period of 1 year. Threshold cycle $(\mathrm{Ct})$ varied $\leq 1$ cycle. Lines indicate median and interquartile range. (B) Circular and NotI linearized plasmid standards differed by approximately $3 \mathrm{Ct}$, leading to an eightfold overestimation of $w \mathrm{OvftsZ/}$ $\mu \mathrm{L}$ using circular plasmid standards. (C) Variation of $w \mathrm{Ovfts} Z$ copies/MF is dependent on the MF count of the preparation. DNA was extracted from $33 \mathrm{MF}$ positive samples and used for qPCR with

triplicates would produce a Wolbachia signal from $1 \mathrm{MF}$, whereas all samples with $\geq 2$ MF provided a positive signal (Fig. 3C). To select a cutoff for quantification, we binned the MF counts as $<10,10-50$, and $>50$ and compared the spread in $95 \% \mathrm{CI}$ of the mean (Fig. 3D). The 10-50 MF samples had a CI spread that was equivalent to that of $>50$ MF ( 71 copies/MF and 82 copies/MF, respectively), while the CI spread from $<10 \mathrm{MF}$ was 266.3 , almost the same as the range. Thus, $\geq 10 \mathrm{MF}$ are needed to quantify the efficacy of antiwolbachial drugs in clinical trials.

Despite the large range of the Wolbachia densities, we expect to be able to evaluate a $2-\log$ reduction under treatment for two reasons. First, the CI spread was smaller using a minimum of $10 \mathrm{MF}$. Second, it was regularly observed in preclinical trials that the range of Wolbachia densities in adult worms becomes smaller after treatment. This was observed with tetracyclines, rifamycins, and chinolones
gBlock® standards. Samples with few MF resulted in a wider range and higher calculated wOvftsZ/MF. Linear regression (blue line) and $95 \%$ confidence bars (red dotted lines) are shown. One sample with 693 MF was omitted from the graph. (D) Determination of a cutoff for quantification was done by comparison of the $95 \%$ confidence intervals (CI) of the means in three different MF-count groups. DNA was extracted from $33 \mathrm{MF}$ positive samples and used for qPCR with $\mathrm{gBlock}{ }^{\circledR}$ standards. The samples were separated into three groups: $<10 \mathrm{MF}, 11-50 \mathrm{MF}$, and $>50 \mathrm{MF}$, and the means and $95 \% \mathrm{CI}$ were calculated (red lines) with GraphPad Prism version 9

(Specht et al. 2018) and the new candidate ABBV-4083 (Hübner et al. 2019), but not with a suboptimal treatment regimen. We are confident to reliably confirm the effectivity of antiwolbachial treatment. In some cases, especially after successful antiwolbachial treatment, the MF from two or more skin snips of a participant could be pooled to achieve the minimum of $10 \mathrm{MF}$ for DNA extraction. However, as a general rule, clinical studies would recruit participants with microfilaridermia high enough to achieve the minimum just using one skin snip.

\section{Conclusion}

The qPCR on skin snip MF provides reliable quantification of Wolbachia in MF and can be used as a surrogate assay for immunohistology of Wolbachia in adult worms in 
nodules extirpated post-treatment that is less invasive, less expensive, and, most importantly, can use easily accessible skin snips. To provide a long-term storable and well-characterized standard for longitudinal monitoring, we selected gBlock ${ }^{\circledR}$ synthetic DNA. The use of the gBlock $®$ standard curve fulfilled the requirements for sensitivity, reproducibility, and stability of the qPCR assay. The qPCR can evaluate Wolbachia densities at more frequent intervals, e.g., during treatment and 2-, 4-, and 6-months post-treatment to monitor Wolbachia depletion longitudinally and gain a more stable assessment of the Wolbachia dynamics to accelerate clinical trials of antiwolbachial therapies.

Supplementary Information The online version contains supplementary material available at https://doi.org/10.1007/s00436-021-07411-5.

Author contribution $\mathrm{AH}, \mathrm{SS}$, UKS, and KP designed the experiments and analyzed the results. AA, PK, SS, CL, BD, MK, and MH did the experiments. FL did the immunofluorescence work. AD and LBD collected the skin biopsies. All authors made substantial contributions to the manuscript in terms of design, experimental work, and intellectual content. All authors read and approved the final manuscript.

Funding Open Access funding enabled and organized by Projekt DEAL. SS received support from the German Center for Infection Research (DZIF, https://www.dzif.de/en) via a clinical leave stipend (TI 07.001_Schlabe_00 and TI 07.001_Schlabe_01). AH, KP, and UKS received funding from the German Center for Infection Research (TI 03.907). The Clinical trials from which the samples were taken were supported by the Bill and Melinda Gates Foundation (DOLF, ISRCTN50035143), BONFOR Research Commission of the Medical Faculty at the University of Bonn and the Commission for Clinical Trials of the University Hospital of Bonn, Germany (MoRiOn, ISRCTN43697583), and a grant from the Liverpool School of Tropical Medicine as part of the A-WOL Consortium funded by the Bill and Melinda Gates Foundation. AH is funded by the Deutsche Forschungsgemeinschaft (DFG, German Research Foundation) under Germany's Excellence Strategy - EXC2151 - 390873048.

Availability of data and material Not applicable.

Code availability Not applicable.

\section{Declarations}

Ethics approval and consent to participate All participants of the prior studies (DOLF, ISRCTN50035143; MoRiOn, ISRCTN43697583; AWOL-Mino, ISRCTN68861628) gave their informed consent prior to study procedures according to local and national laws and in line with the requirements of Good Clinical Practice. All participants gave their informed consent for their samples to be biobanked and used for biomarker analysis. The informed consent included the consent to participate and consent to publication. The animal work was approved according to the rules of the animal welfare and protection act and national laws, specifically in accordance with the European Union Directive 2010/63/EU. Experiments were carried out after approval by the Landesamt fürNatur, Umwelt und Verbraucherschutz, Cologne, Germany (AZ 84-02.04.2015.A507).

Conflict of interest The authors declare no competing interests.
Open Access This article is licensed under a Creative Commons Attribution 4.0 International License, which permits use, sharing, adaptation, distribution and reproduction in any medium or format, as long as you give appropriate credit to the original author(s) and the source, provide a link to the Creative Commons licence, and indicate if changes were made. The images or other third party material in this article are included in the article's Creative Commons licence, unless indicated otherwise in a credit line to the material. If material is not included in the article's Creative Commons licence and your intended use is not permitted by statutory regulation or exceeds the permitted use, you will need to obtain permission directly from the copyright holder. To view a copy of this licence, visit http://creativecommons.org/licenses/by/4.0/.

\section{References}

Albers AC (2011) Evaluation of Wolbachia depletion after antibiotic treatment using real-time PCR in onchocerciasis and lymphatic filariasis, and analysis of genetic associations in lymphatic filariasis. Rheinische Friedrich Wilhelms Universität Bonn

Batsa-Debrah L et al (2017) Doxycycline for the treatment of onchocerciasis: a daily dose of $100 \mathrm{mg}$ for 6 weeks shows reduction of fertile female Onchocera volvulus equivalent to $200 \mathrm{mg} / \mathrm{d}$ (531). Paper presented at the 66th Annual Meeting ASTMH, Baltimore, Maryland, USA, November 5-9, 2017

Batsa Debrah L et al (2020) Comparison of repeated doses of ivermectin versus ivermectin plus albendazole for the treatment of onchocerciasis: a randomized, open-label, clinical trial. Clin Infect Dis 71(4):933-943. https://doi.org/10.1093/cid/ciz889

Büttner DW, Wanji S, Bazzocchi C, Bain O, Fischer P (2003) Obligatory symbiotic Wolbachia endobacteria are absent from Loa loa. Filaria J 2(1):10. https://doi.org/10.1186/1475-2883-2-10

Conte J, Potoczniak MJ, Tobe SS (2018) Using synthetic oligonucleotides as standards in probe-based qPCR. Biotechniques 64(4):177-179. https://doi.org/10.2144/btn-2018-2000

Debrah AY et al (2015) Doxycycline leads to sterility and enhanced killing of female Onchocerca volvulus worms in an area with persistent microfilaridermia after repeated ivermectin treatment: a randomized, placebo-controlled, double-blind trial. Clin Infect Dis 61(4):517-526. https://doi.org/10.1093/cid/civ363

Fattouh N, Cazevieille C, Landmann F (2019) Wolbachia endosymbionts subvert the endoplasmic reticulum to acquire host membranes without triggering ER stress. PLoS Negl Trop Dis 13(3):e0007218. https://doi.org/10.1371/journal.pntd.0007218

Hoerauf A, Mand S, Adjei O, Fleischer B, Buttner DW (2001) Depletion of Wolbachia endobacteria in Onchocerca volvulus by doxycycline and microfilaridermia after ivermectin treatment. Lancet 357(9266):1415-1416. https://doi.org/10.1016/S0140-6736(00) 04581-5

Hoerauf A et al (2008) Wolbachia endobacteria depletion by doxycycline as antifilarial therapy has macrofilaricidal activity in onchocerciasis: a randomized placebo-controlled study. Med Microbiol Immunol 197(3):295-311. https://doi.org/10.1007/ s00430-007-0062-1

Hong WD et al (2019) AWZ1066S, a highly specific anti-Wolbachia drug candidate for a short-course treatment of filariasis. Proc Natl Acad Sci U S A 116(4):1414-1419. https://doi.org/10.1073/pnas. 1816585116

Hou Y, Zhang H, Miranda L, Lin S (2010) Serious overestimation in quantitative PCR by circular (supercoiled) plasmid standard: microalgal PCNA as the model gene. PLoS ONE 5(3):e9545. https://doi.org/10.1371/journal.pone.0009545 
Hübner MP et al (2019) In vivo kinetics of Wolbachia depletion by ABBV-4083 in L. sigmodontis adult worms and microfilariae. PLoS Negl Trop Dis 13(8):e0007636. https://doi.org/10.1371/ journal.pntd.0007636

Hübner MP et al (2020) Oxfendazole mediates macrofilaricidal efficacy against the filarial nematode Litomosoides sigmodontis in vivo and inhibits Onchocerca spec. motility in vitro. PLoS Negl Trop Dis 14(7):e0008427. https://doi.org/10.1371/journal.pntd.0008427

Klarmann-Schulz U et al (2017) Comparison of doxycycline, minocycline, doxycycline plus albendazole and albendazole alone in their efficacy against onchocerciasis in a randomized, open-label, pilot trial. PLoS Negl Trop Dis 11(1):e0005156. https://doi.org/ 10.1371/journal.pntd.0005156

Krücken J et al (2021) Development of emodepside as possible adulticidal treatment for human onchocerciasis - the fruit of a successful industrial-academic collaboration. PLoS Pathog 17(7):e1009682. https://doi.org/10.1371/journal.ppat.1009682

Landmann F, Foster JM, Slatko BE, Sullivan W (2012) Efficient in vitro RNA interference and immunofluorescence-based phenotype analysis in a human parasitic nematode Brugia Malayi. Parasit Vectors 5:16. https://doi.org/10.1186/1756-3305-5-16

Lin CH, Chen YC, Pan TM (2011) Quantification bias caused by plasmid DNA conformation in quantitative real-time PCR assay. PLoS ONE 6(12):e29101. https://doi.org/10.1371/journal.pone.0029101

Martinez-Palomo A, Martinez-Baez M (1977) Ultrastructure of the microfilaria of Onchocerca volvulus from Mexico. J Parasitol 63(6):1007-1018. https://doi.org/10.2307/3279835

Serbus LR et al (2012) A cell-based screen reveals that the albendazole metabolite, albendazole sulfone, targets Wolbachia. PLoS Pathog 8(9):e1002922. https://doi.org/10.1371/journal.ppat.1002922

Specht $\mathrm{S}$ et al (2018) Combinations of registered drugs reduce treatment times required to deplete Wolbachia in the Litomosoides sigmodontis mouse model. PLoS Negl Trop Dis 12(1):e0006116. https://doi.org/10.1371/journal.pntd.0006116

Specht S, Hoerauf A, Adjei O, Debrah A, Buttner DW (2009) Newly acquired Onchocerca volvulus filariae after doxycycline treatment. Parasitol Res 106(1):23-31. https://doi.org/10.1007/ s00436-009-1624-5

Taylor MJ, et al. (2019) Preclinical development of an oral anti-Wolbachia macrolide drug for the treatment of lymphatic filariasis and onchocerciasis. Sci Transl Med 11(483), https://doi.org/10. 1126/scitranslmed.aau2086

Turner HC, Churcher TS, Walker M, Osei-Atweneboana MY, Prichard RK, Basanez MG (2013) Uncertainty surrounding projections of the long-term impact of ivermectin treatment on human onchocerciasis. PLoS Negl Trop Dis 7(4):e2169. https://doi.org/10.1371/ journal.pntd.0002169

Turner JD et al (2006) A randomized, double-blind clinical trial of a 3 -week course of doxycycline plus albendazole and ivermectin for the treatment of Wuchereriabancrofti infection. Clin Infect Dis 42(8):1081-1089. https://doi.org/10.1086/501351

von Geldern TW et al (2019) Discovery of ABBV-4083, a novel analog of Tylosin A that has potent anti-Wolbachia and anti-filarial activity. PLoS Negl Trop Dis 13(2):e0007159. https://doi.org/10.1371/ journal.pntd.0007159

WHO (2020) Elimination of human onchocerciasis: progress report, 2019-2020. Wkly Epidemiol Rec 95:545-556

Publisher's note Springer Nature remains neutral with regard to jurisdictional claims in published maps and institutional affiliations. 